\title{
Development and Validation of a Prediction Model for 5-Year Risk of Kidney Dysfunction in Patients with Hypertension and Glucose Metabolism Disorder
}

\author{
Mengyue Lin $\mathbb{D}^{1,2}$, Mulalibieke Heizhati', Lin Gan $\mathbb{D}^{1,2}$, Ling Yao', Wenbo Yang', Mei Li', Jing Hong', \\ Zihao Wu', Hui Wang ${ }^{1,2}$, Nanfang Li $\mathbb{B}^{\prime}$ \\ 'Hypertension Center of People's Hospital of Xinjiang Uygur Autonomous Region; Xinjiang Hypertension Institute; National Health Committee Key \\ Laboratory of Hypertension Clinical Research; Key Laboratory of Xinjiang Uygur Autonomous Region "Hypertension Research Laboratory"; Xinjiang \\ Clinical Medical Research Center for Hypertension (Cardio-Cerebrovascular) Diseases, Urumqi, People's Republic of China; ${ }^{2}$ Xinjiang Medical \\ University, Urumqi, Xinjiang, People's Republic of China \\ Correspondence: Nanfang Li, Email Inanfang20I6@sina.com
}

Purpose: Patients with hypertension and glucose metabolism disorder (GMD) are at high risk of developing kidney dysfunction (KD). Therefore, we aimed to develop a nomogram for predicting individuals' 5-year risk of KD in hypertensives with GMD.

Patients and Methods: In total, 1961 hypertensives with GMD were consecutively included. Baseline data were extracted from medical electronic system, and follow-up data were obtained using annual health check-ups or hospital readmission. KD was defined as estimated glomerular filtration rate (eGFR) $<60 \mathrm{~mL} / \mathrm{min} / 1.73 \mathrm{~m}^{2}$. Subjects were randomly divided into training and validation sets with a ratio of 7 to 3 . Least absolute shrinkage and selection operator method was used to identify potential predictors. Cox proportional hazard model was applied to build a nomogram for predicting KD risk. The discriminative ability, calibration and usefulness of the model were evaluated. The prediction model was verified by internal validation.

Results: During the follow-up of 5351 person-years with a median follow-up of 32 (range: 3-91) months, 130 patients developed KD. Age, sex, ethnicity, hemoglobin A1c, uric acid, and baseline eGFR were identified as significant predictors for incident KD and used for establishing nomogram. The prediction model displayed good discrimination with C-index of 0.770 (95\% CI: 0.712-0.828) and 0.763 (95\% CI: 0.704-0.823) in training and validation sets, respectively. Calibration curve indicated good agreement between the predicted and actual probabilities. The decision curve analysis demonstrated that the model was clinically useful.

Conclusion: The prediction nomogram, including six common easy-to-obtain factors, shows good performance for predicting 5-year risk of KD in hypertensives with GMD. This quantitative tool could help clinicians, and even primary care providers, recognize potential KD patients early and make strategy for prevention and management.

Keywords: kidney dysfunction, nomogram, hypertension, diabetes, prediction

\section{Introduction}

Chronic kidney disease (CKD) has been shown to be closely related to cardiovascular events, end-stage renal disease and premature death. ${ }^{1}$ In 2013, there were 956,200 deaths directly attributed to CKD worldwide. ${ }^{2}$ Due to the asymptomatic nature in early-stage, most of the patients are already processed to an irreversible stage at the time of detection for CKD, usually with complications, and some of them even need renal replacement therapy. ${ }^{3}$ It has been reported that the global prevalence of CKD is $13.4 \%,{ }^{4}$ with approximately 700 million people who have reduced glomerular function in $2017 .^{5}$ Moreover, incidence of CKD has been increasing, largely due to an ongoing epidemic of metabolic disorders such as hypertension and glucose metabolism disorder (GMD), both of which have been shown to be leading causes of CKD in developed countries, ${ }^{6}$ and are becoming leading causes in developing countries where hypertension and diabetes have risen rapidly in the past 20-30 years. ${ }^{7}$ Reportedly, one-third patients with diabetes and one-fifth patients with hypertension may have CKD in America. ${ }^{8}$ More than $40 \%$ diabetic patients in Japan are affected by kidney damage. ${ }^{9}$ Also, 
approximately $40 \%$ of CKD is associated with hypertension and diabetes among hospitalized patients in China. ${ }^{10}$ In addition, as an important component of GMD, prevalence of prediabetes is as high as $38.1 \%$ among Chinese adults, ${ }^{11}$ and is also associated with adverse outcomes. ${ }^{12,13}$

Kidney dysfunction (KD) is an important component of CKD. Given that hypertension and GMD are risk factors for each other and often co-exist, ${ }^{14}$ we recently conducted a population-based study in China and observed that the prevalence of KD in patients with both hypertension and diabetes is higher than in those with either one alone, ${ }^{15}$ indicating a high risk for KD in this population. However, controlling blood pressure and glucose does not seem to have achieved the goal of preventing KD, possibly in part by failing to accurately predict high-risk individuals. In addition, some patients have irreversible kidney damage by the time KD is detected, also due to a failure to recognize it early. Recently, several biomarkers, such as homocysteine and osteoprotegerin, are shown to be involved in organ damage in patients with hypertension or CKD. ${ }^{16-18}$ However, the benefits of intervention in such markers for renal function have yet to be evaluated. Given the adverse outcomes of $\mathrm{KD}$, identifying and predicting the risk is important for preventing or delaying the occurrence and development of KD. Moreover, since KD and cardiovascular diseases share common risk factors, the prediction of KD may also have benefits in preventing multiple morbidities. ${ }^{19}$

Several predictive tools for kidney diseases have been established. ${ }^{20-23}$ However, no studies have focused on specific population group with both hypertension and GMD. Intervention in the early stages can effectively prevent the onset and progression of kidney disease. ${ }^{24}$ Therefore, we aimed to develop and validate a predictive nomogram based on common variables to predict individuals' risk of incident $\mathrm{KD}$ in this specific patient population.

\section{Methods}

\section{Study Population}

Participants in the current study were patients admitted to Hypertension Center of People's Hospital of Xinjiang Uygur Autonomous Region from January 2012 to May 2019. Patients who aged 18 years or older, with hypertension and GMD, were screened through an electronic medical record system. Exclusion criteria were diagnosis of secondary hypertension (including primary aldosterone, adrenal tumor, Cushing syndrome, pheochromocytoma, and polycystic ovary syndrome), history of cardiovascular events within the last 3 months (including myocardial infarction, heart failure, stroke, unstable angina, coronary revascularization and coronary bypass operation), or malignant tumor. At this stage, 2946 patients were eligible. Among them, those with CKD at baseline $(n=410)$, with any missing data $(n=105)$, and who did not have follow-up data $(\mathrm{n}=470)$ were excluded. Therefore, 1961 individuals were finally included in the statistical analysis. The study was conducted in accordance with the Declaration of Helsinki and approved by the Ethics Committee at People's Hospital of Xinjiang Uygur Autonomous Region. All participants or their legal representatives signed written consent forms.

\section{Data Collection}

Baseline data were extracted from medical electronic system, including age, sex, body mass index (BMI), blood pressure (BP), ethnicity (Han, Uyghur or others), marital status (married or single/separated), duration of hypertension, types of GMD (pre-diabetes or diagnosed diabetes), cigarette consumption (yes or no), alcohol intake (yes or no), fasting plasma glucose (FPG), hemoglobin A1c (HbA1c), total cholesterol (TC), triglyceride (TG), high-density lipoprotein cholesterol (HDL-C), low-density lipoprotein cholesterol (LDL-C), blood urea nitrogen (BUN), uric acid (UA), serum creatinine (Scr), serum potassium, anti-hypertensive drugs, history of cardiovascular diseases.

\section{Definition of Diseases}

Hypertension is defined as systolic BP (SBP) $\geq 140 \mathrm{mmHg}$ and/or diastolic BP (DBP) $\geq 90 \mathrm{mmHg}$, or under antihypertensive therapy. GMD includes impaired fasting glucose (IFG), impaired glucose tolerance (IGT), and diagnosed diabetes mellitus (DM). IFG is defined if a FPG ranges from 6.1 to $<7.0 \mathrm{mmol} / \mathrm{L}$ whereas 2-hour glucose is $<7.8 \mathrm{mmol} / \mathrm{L}$; IGT is defined if 2-hour glucose ranged from 7.8 to $<11.1 \mathrm{mmol} / \mathrm{L}$; DM is defined if there is a previously confirmed diagnosis, or FPG was $\geq 7.0 \mathrm{mmol} / \mathrm{L}$, or 2 -hour glucose was $\geq 11.1 \mathrm{mmol} / \mathrm{L}{ }^{25}$ Prediabetes include $\mathrm{IFG}$ and IGT. 
Estimated glomerular filtration rate (eGFR) is calculated by the Chronic Kidney Disease Epidemiology Collaboration (CKD-EPI) equation. ${ }^{26} \mathrm{KD}$ is defined as eGFR $<60 \mathrm{~mL} / \mathrm{min} / 1.73 \mathrm{~m}^{2}$.

\section{Follow-Up and Outcome}

The outcome of the current study was a new-onset KD during follow up. Follow-up data were obtained using annual health check-ups or hospital readmission. Examination time of 3 months or longer after baseline was considered to be valid data. If a participant experienced the outcome more than once during follow-up, only the first outcome was used for analysis. For those without KD events during follow-up, the data of the last follow-up was included in the analysis.

\section{Statistical Analyses}

The study is consistent with the transparent report of the multivariable predictive model of individual prognosis or diagnosis (TRIPOD): the TRIPOD statement. ${ }^{27}$ Continuous variables were presented as mean \pm standard deviation or median (interquartile range) and compared between groups using student $t$ test or non-parametric Mann-Whitney $U$-test. Categorical variables were summarized as number and percentage and compared between groups using Chi-square test. The processes for development and validation of the predictive model were as follows. First, 1961 participants were randomly divided with a ratio of 7 to 3 into a training set $(n=1372)$ and a validation set $(n=589)$, for development and validation of the model, respectively. Second, the training set was analyzed using the least absolute shrinkage and selection operator (LASSO) regression to identify independent predictive features with nonzero coefficients. ${ }^{28,29}$ LASSO regression is a method to simplify high-dimensional data and is often used to select features for developing prediction model. Third, variables selected by LASSO regression were included in the univariable and multivariable Cox regression analysis in the training set, and the results were presented as hazard ratio (HR) and 95\% confidence interval (95\% CI). Fourth, the performance of the prediction model was evaluated on the training and validation sets. C-index and area under the receiver operating characteristic curve (AUC) were used for evaluating the discrimination of the nomogram. Calibration, which refers to the consistency between the predicted risk and the actual risk, was visualized by calibration curve plot and tested by Hosmer-Lemeshow test. Decision curve analysis was conducted to determine the clinical usefulness of the nomogram, which quantified the net benefits at different threshold probabilities in the study. Bootstraps for 1000 resamples were applied on the C-index, AUC, calibration curve, and decision curve analysis to reduce the overfitting deviation. Finally, the cumulative incidence of $\mathrm{KD}$ between high- and low-risk groups according to the cut-off value of $50 \%$ of predictive risk was estimated using the Kaplan-Meier method and compared using Log rank test. Statistical analyses were performed using R software version 4.0.3 (https://www.R-project.org) and SPSS version 25.0 for Windows (SPSS Inc., Chicago, Illinois, USA).

\section{Results}

\section{Characteristics of the Study Subjects}

A total of 1961 patients with hypertension and GMD were finally included. The mean age and BMI were $55.4 \pm 11.0$ years and $28.1 \pm 3.9 \mathrm{~kg} / \mathrm{m}^{2}$, respectively. $844(43.0 \%)$ were women, $1133(57.8 \%)$ had diagnosed DM, and $634(32.3 \%)$ had history of cardiovascular disease at baseline. During the total follow-up of 5351 person-years with a median followup of 32 (range: 3-91) months, 130 (6.6\%) participants developed KD with an incidence of 24.3/1000 person-years. The baseline characteristics of the subjects are shown in Table 1. There were no significant differences observed between the training and validation sets.

\section{Characteristics Selection and Cox Regression Analysis}

Six potential variables out of 23 factors were identified by LASSO analysis (Figure S1), including age, sex, ethnicity, $\mathrm{HbA1c}$, UA and baseline eGFR, which were considered to be independent predictors of KD in this specific patient population. Results of univariate and multivariate Cox regression analysis are given in Table 2. 
Table I Characteristics of the Subjects in Training and Validation Sets

\begin{tabular}{|c|c|c|c|c|}
\hline Characteristic & Total $(n=1961)$ & Training Set $(n=1372)$ & Validation Set $(n=589)$ & $P$ value \\
\hline Age (years) & $55.4 \pm 11.0$ & $55.2 \pm 10.9$ & $55.7 \pm 11.2$ & 0.362 \\
\hline Female, n (\%) & $844(43.0)$ & $593(43.2)$ & $25 I(42.6)$ & 0.803 \\
\hline \multicolumn{5}{|l|}{ Ethnicity, n (\%) } \\
\hline Han & II 58 (59.1) & $818(59.6)$ & $340(57.7)$ & 0.569 \\
\hline Uyghur & $521(26.6)$ & $355(25.9)$ & $166(28.2)$ & \\
\hline Others & $282(14.1)$ & $199(14.5)$ & $83(4.2)$ & \\
\hline Smoking, n (\%) & $575(29.3)$ & $40 \mathrm{I}(29.2)$ & $174(29.5)$ & 0.889 \\
\hline Drinking, n (\%) & $527(26.9)$ & $380(27.7)$ & $147(25.0)$ & 0.210 \\
\hline Body mass index $\left(\mathrm{kg} / \mathrm{m}^{2}\right)$ & $28.1 \pm 3.9$ & $28.1 \pm 3.9$ & $28.2 \pm 4.0$ & 0.488 \\
\hline Systolic blood pressure $(\mathrm{mmHg})$ & $148.5 \pm 21.2$ & $147.9 \pm 20.7$ & $149.9 \pm 22.3$ & 0.061 \\
\hline Diastolic blood pressure $(\mathrm{mmHg})$ & $88.0 \pm 14.8$ & $87.7 \pm 14.7$ & $88.6 \pm 14.9$ & 0.244 \\
\hline Duration of HTN (years) & $7.0(2.0,12.0)$ & $7.0(2.0,12.0)$ & $7.0(2.0,12.0)$ & 0.602 \\
\hline \multicolumn{5}{|l|}{ Anti-hypertension drugs, n (\%) } \\
\hline 1 & $646(32.9)$ & $446(32.5)$ & $200(34.0)$ & 0.169 \\
\hline 2 & $776(39.6)$ & $561(40.9)$ & $215(36.5)$ & \\
\hline$\geq 3$ & $539(27.5)$ & $365(26.6)$ & $174(29.5)$ & \\
\hline \multicolumn{5}{|l|}{ Type of GMD, n (\%) } \\
\hline Prediabetes & $828(42.2)$ & $572(41.7)$ & $256(43.5)$ & 0.466 \\
\hline Diagnosed diabetes & II $33(57.8)$ & $800(58.3)$ & $333(56.5)$ & \\
\hline History of CVD, n (\%) & $634(32.3)$ & $444(32.4)$ & $190(32.3)$ & 0.964 \\
\hline Fasting plasma glucose $(\mathrm{mmol} / \mathrm{L})$ & $6.2 \pm 2.3$ & $6.2 \pm 2.3$ & $6.2 \pm 2.2$ & 0.511 \\
\hline HbAlc (\%) & $6.9 \pm 1.3$ & $6.9 \pm 1.3$ & $6.9 \pm 1.3$ & 0.927 \\
\hline Total cholesterol $(\mathrm{mmol} / \mathrm{L})$ & $4.42 \pm 1.05$ & $4.42 \pm 1.04$ & $4.42 \pm 1.10$ & 0.930 \\
\hline Total triglyceride $(\mathrm{mmol} / \mathrm{L})$ & $2.15 \pm 1.93$ & $2.13 \pm 1.87$ & $2.19 \pm 2.07$ & 0.575 \\
\hline HDL-C (mmol/L) & $0.97 \pm 0.23$ & $0.97 \pm 0.24$ & $0.97 \pm 0.21$ & 0.502 \\
\hline LDL-C (mmol/L) & $2.61 \pm 0.85$ & $2.62 \pm 0.85$ & $2.59 \pm 0.86$ & 0.474 \\
\hline Baseline eGFR $\left(\mathrm{mL} / \mathrm{min} / \mathrm{I} .73 \mathrm{~m}^{2}\right)$ & $99.3 \pm 13.7$ & $99.5 \pm 13.7$ & $98.8 \pm 13.6$ & 0.335 \\
\hline Blood urea nitrogen (mmol/L) & $5.07 \pm 1.35$ & $5.03 \pm 1.32$ & $5.14 \pm 1.41$ & 0.140 \\
\hline Uric acid $(\mu \mathrm{mol} / \mathrm{L})$ & $331.5 \pm 84.5$ & $330.3 \pm 84.8$ & $334.2 \pm 83.8$ & $0.34 I$ \\
\hline Serum potassium $(\mathrm{mmol} / \mathrm{L})$ & $3.67 \pm 0.28$ & $3.67 \pm 0.28$ & $3.68 \pm 0.29$ & 0.400 \\
\hline Incident KD, n (\%) & $130(6.6)$ & $85(6.2)$ & $45(7.6)$ & 0.238 \\
\hline
\end{tabular}

Notes: Data are presented as means \pm standard deviation, median (interquartile) or number (percentage).

Abbreviations: HTN, hypertension; GMD, glucose metabolic disorders; CVD, cardiovascular disease; FPG, fasting plasma glucose; HDL-C, high density lipoprotein cholesterol; LDL-C, low density lipoprotein cholesterol; eGFR, estimated glomerular filtration rate; KD, kidney dysfunction.

\section{Development of a Predictive Nomogram}

Nomogram, a quantitative and convenient tool, was built based on the multivariable Cox model. As shown in Figure 1, baseline eGFR corresponded to the strongest association with incident KD; HbA1c and UA also showed strong relation with KD risk. To estimate an individual's 5-year risk of incident $\mathrm{KD}$, each predictor's point is determined according to the top points scale. Then, the points of the 6 variables were summed. Finally, the sum was located on the total points scale and projected vertically on the lowest axis. For example, in patients with hypertension and GMD, assuming a Hanethnic women (18 points) aged 50 years old ( 23 points) with HbAlc of $8 \%$ (42 points), UA of $400 \mu \mathrm{mol} / \mathrm{L}$ (34 points), eGFR of $100 \mathrm{~mL} / \mathrm{min} / 1.73 \mathrm{~m}^{2}$ (55 points), the 5 -year probability of developing $\mathrm{KD}$ is estimated to be $85 \%$.

\section{Performance of the Nomogram}

The C-index was 0.770 (95\% CI: 0.712-0.828) and 0.763 (95\% CI: $0.704-0.823$ ) in the training and validation sets, respectively. Similarly, the AUC of the predicted nomogram was 0.788 and 0.775 (Figure $2 \mathrm{a}$ and $\mathrm{b}$ ), indicating that the model contained good ability of discrimination and prediction. Calibration curves suggested a good agreement between the actual probability and the predicted probability (Figure 3a for the training set and $\mathrm{b}$ for the validation set), and the results were verified by Hosmer-Lemeshow test $(P=0.615$ in the training set, $P=0.587$ in the validation set). The 
Table 2 Cox Regression Analysis for Selecting Factors Associated with KD

\begin{tabular}{|c|c|c|c|c|}
\hline \multirow[t]{2}{*}{ Variables } & \multicolumn{2}{|c|}{ Univariable } & \multicolumn{2}{|c|}{ Multivariable } \\
\hline & HR (95\% Cl) & $P$ value & HR (95\% CI) & $P$ value \\
\hline Age & I.053 (I.03।, I.074) & $<0.001$ & $1.026(1.003,1.050)$ & 0.027 \\
\hline Female & $1.839(1.195,2.830)$ & 0.006 & $1.826(1.132,2.946)$ & 0.014 \\
\hline \multicolumn{5}{|l|}{ Ethnicity } \\
\hline Han & \multicolumn{2}{|c|}{ Reference } & \multicolumn{2}{|c|}{ Reference } \\
\hline Uyghur & $1.090(0.644,1.843)$ & 0.748 & $1.134(0.662,1.943)$ & 0.646 \\
\hline Others & $1.876(1.098,3.206)$ & 0.021 & $1.979(1.150,3.406)$ & 0.014 \\
\hline $\mathrm{HbAlc}$ & I.24I (I.105, I.395) & $<0.001$ & $1.324(1.168,1.501)$ & $<0.001$ \\
\hline Uric acid & $1.001(0.998,1.003)$ & $0.47 \mid$ & $1.003(1.000,1.006)$ & 0.035 \\
\hline eGFR & $0.959(0.945,0.973)$ & $<0.001$ & $0.963(0.946,0.98 I)$ & $<0.001$ \\
\hline
\end{tabular}

Notes: The above variables were selected based on LASSO analysis.

Abbreviations: eGFR, estimated glomerular filtration rate; KD, kidney dysfunction; HbAlc, glycated hemoglobin; HR, hazard ratio.

decision curve analysis showed that using this nomogram to predict the KD risk yields more net benefit than the intervention-for-all-patients scheme (Figure 4). Based on the constructed model, patients were divided into high- or lowrisk groups according to the cut-off value of $50 \%$ of predicted risk. Kaplan-Meier curve analysis showed that there was a significant difference in the cumulative incidence of KD between high- and low-risk groups (Figure 5).

\section{Discussion}

The present cohort, including hypertensives with GMD who had preserved renal function at baseline, show that the incidence of $\mathrm{KD}$ is approximately $2.5 \%$ per year. In this specific population at high-risk for $\mathrm{KD}$, age, sex, ethnicity,

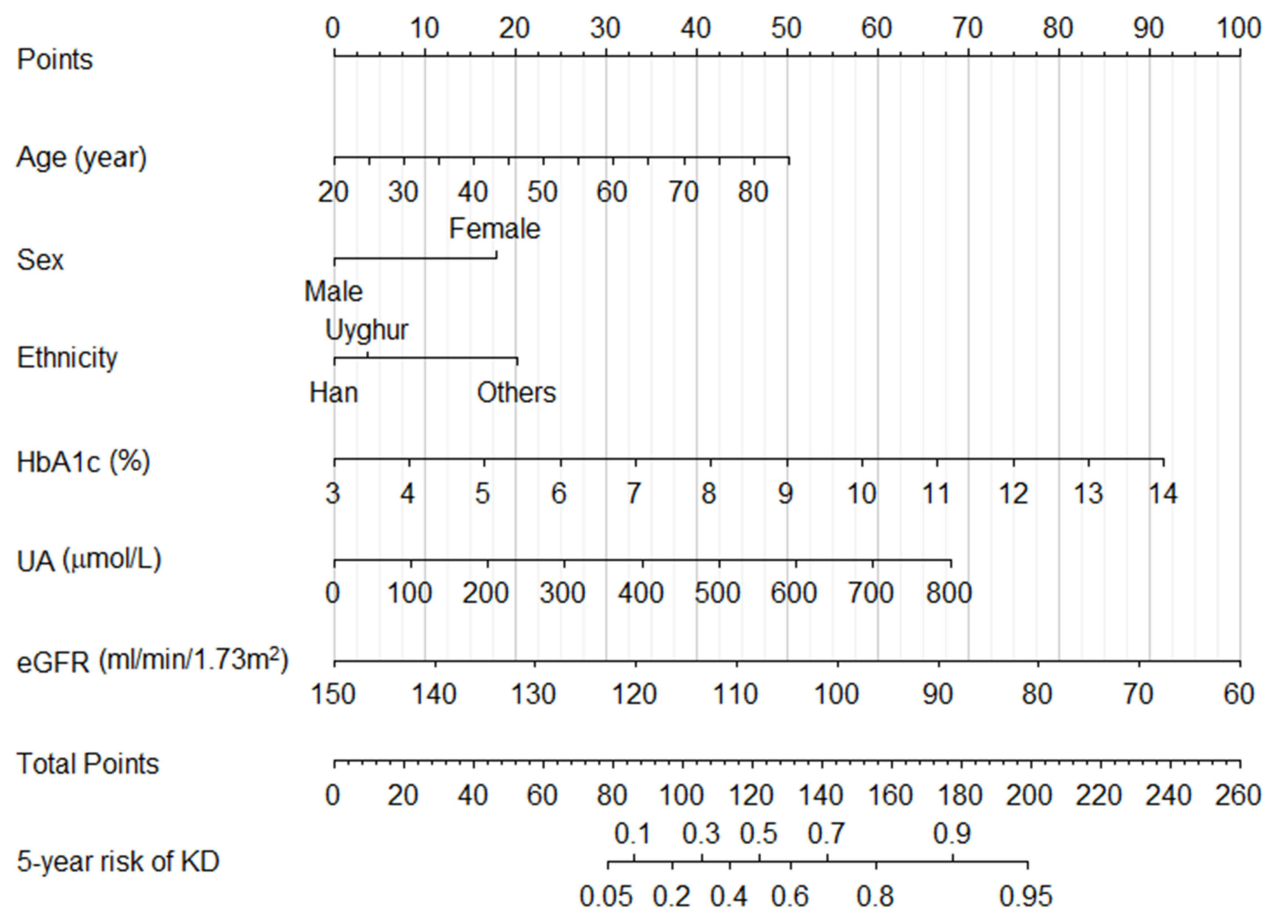

Figure I Nomogram for predicting the 5-year risk of KD in patient with hypertension and glucose metabolic disorders. To use the nomogram, find the position of each variable on the corresponding axis. A vertical line was drawn from that value to the top points scale to determine the number of points that were assigned by that variable value. Then, the points from each variable value were added. Finally, draw a line from the total points axis to estimate the 5-year risk of KD at the lowest line of the nomogram.

Abbreviations: KD, kidney dysfunction; SBP, systolic blood pressure; DM, diabetes mellitus; UA, uric acid; eGFR, estimated glomerular filtration rate. 

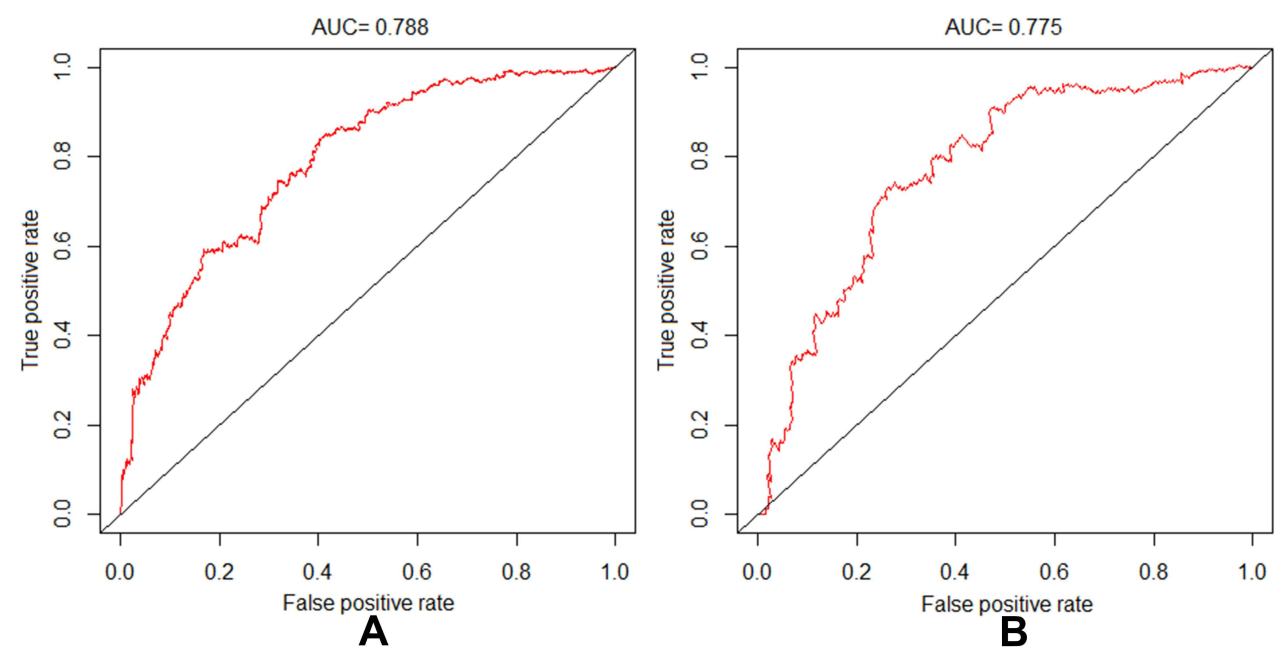

Figure 2 Receiver-operating characteristic (ROC) curves of the models in derivation $(\mathbf{A})$ and validation $(\mathbf{B})$ sets. AUC: area under the curve (bootstrap resampling times = 1000).

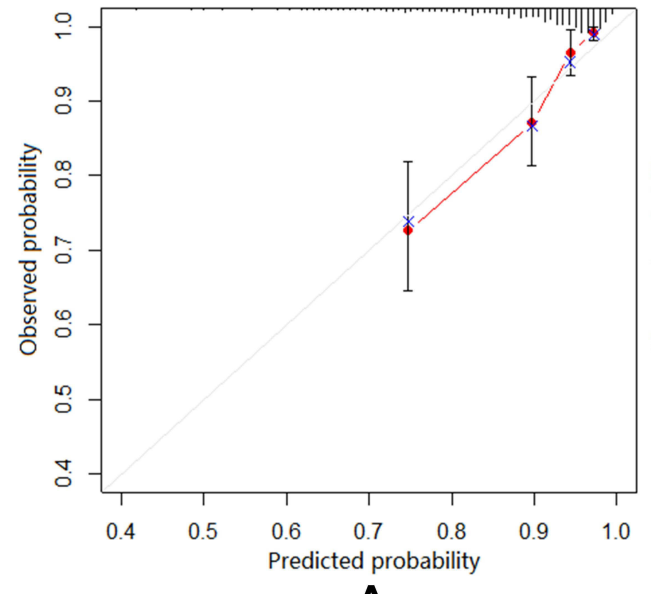

A

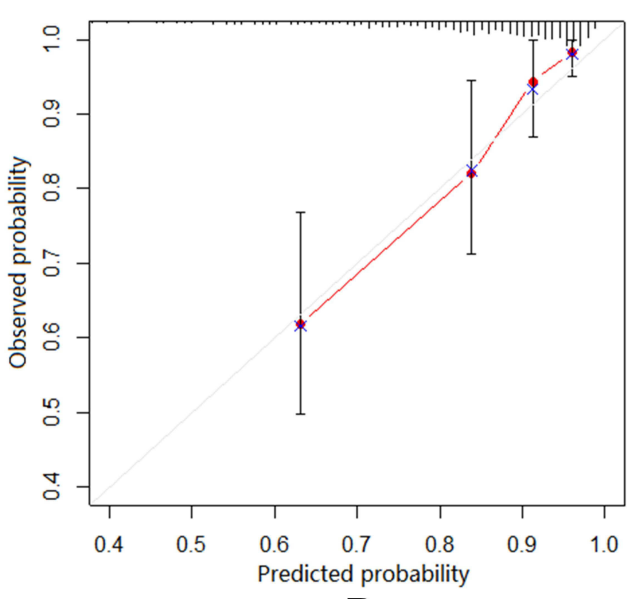

B

Figure 3 Calibration curves of the kidney dysfunction prediction models. (A) Calibration curve of the nomogram in the training set. (B) Calibration curve of the nomogram in the validation set. The diagonal line represents a perfect prediction by an ideal model. The red line represents the performance of the nomogram, of which a closer fit to the diagonal line represents a better prediction. The vertical bar represents the $95 \%$ range of the selected points (bootstrap resampling times $=1000$ ).

baseline HbA1c, UA and eGFR are significantly and independently associated with the development of KD. The prediction model based on the above six factors shows good discrimination, excellent calibration, and satisfactory validity and clinical utility, indicating good performance for predicting 5-years risk of incident KD.

Hypertension and GMD are risk factors for KD. Prevalence of KD in patients with both hypertension and DM is higher than that in patients with each one alone. ${ }^{15,30}$ The present study with longitudinal observation shows that the annual incidence of $\mathrm{KD}$ is $\approx 2.5 \%$ in this patient population, which is higher than in the general population. ${ }^{31}$ The results are consistent with a recent systematic review of 71 studies, which reports that the annual incidence of $\mathrm{CKD}$ is $1.9 \%$ to 4.3\% among DM patients. ${ }^{32}$ Hypertension and GMD have been strongly associated with macrovascular and microvascular diseases. ${ }^{33,34}$ However, few studies have focused specifically on this patient group with high risk for KD. In line with previous studies, older age and female gender are risk factors for KD in our study. ${ }^{35,36}$ Ethnicity is also associated with incident KD. Different ethnicity may stand for differences in lifestyle and dietary habits and even differences in risk factors, which may serve partly as the reasons. For example, high prevalence and poor management of hypertension in some ethnicities, such as Kazakh, Tajik, and Mongolian, may also play important roles in this process. ${ }^{37,38}$ 


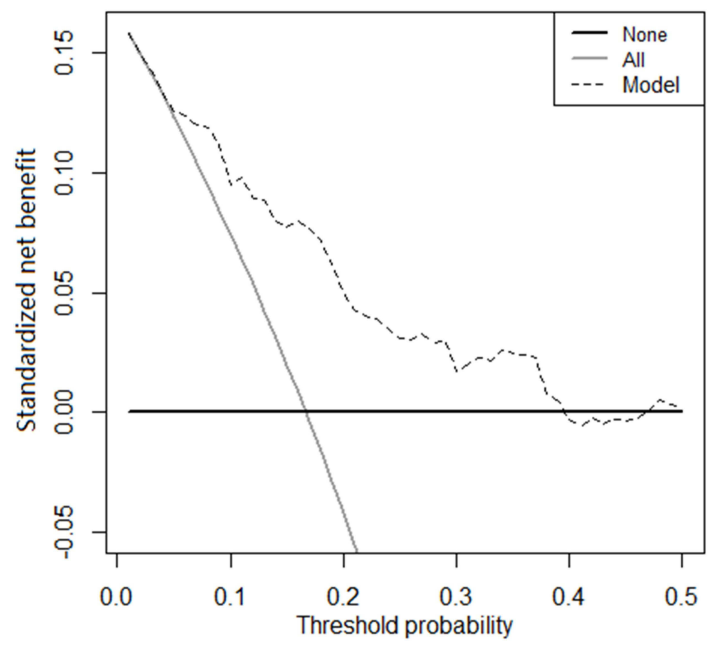

Figure 4 Decision curve analysis of the nomogram for predicting KD. The $x$-axis indicates the threshold probability. The dotted line represents the prediction nomogram. The black line displays the net benefit under the assumption that all patients are non-KD. The gray line displays the net benefit under the assumption that all patients are KD (bootstrap resampling times $=1000$ ).

Abbreviation: KD, kidney dysfunction.

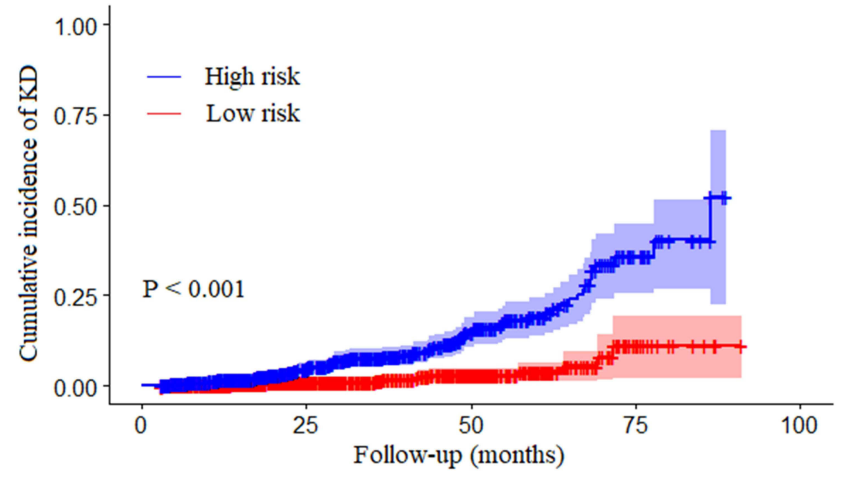

Number at risk

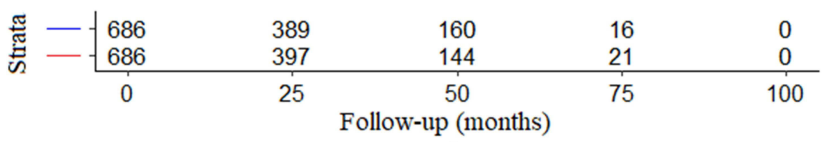

A

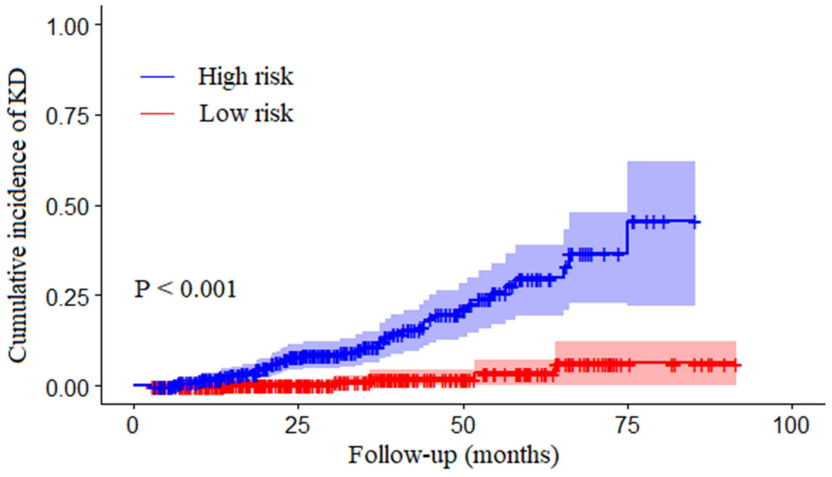

Number at risk

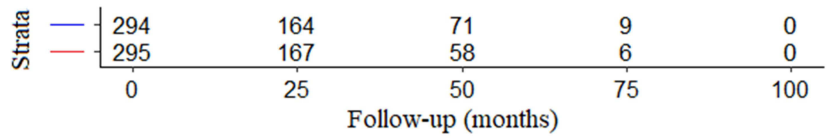

B

Figure 5 Kaplan-Meier curves of cumulative incidence of kidney dysfunction in training set (A) and validation set (B). High- (blue) and low-risk (red) group stratification were based on the predictor derived from nomogram prediction model. Shadow represents the $95 \%$ confidence interval.

With regard to the controllable factors, baseline eGFR serves as the strongest predictor for incident KD in this study, consistent with other cohorts conducted in both general population and high-risk groups for KD. They show a close association between baseline eGFR and the development of CKD. ${ }^{39,40}$ A decrease in GFR affects BP levels, which in turn worsens kidney function, creating a vicious cycle that eventually leads to irreversible kidney damage. ${ }^{41}$ Compared with non-DM participants, DM patients are shown to have a higher risk for developing CKD. Our study shows that glucose is also closely associated with incident KD in hypertensives with GMD. In this regard, as an indicator of longterm blood glucose level, HbA1c is more predictive of incident KD than FPG. Interestingly, neither BP level nor hypertension duration is selected into the prediction model in our study. DM has been shown to accelerate the progression of renal function decline two-times over hypertension. ${ }^{42,43}$ Thus, the impact of hypertension on kidney damage may be covered up by the co-existence of DM. However, management of hypertension and controlling BP level should be taken seriously, given the well-proven association between high BP and kidney impairment. Furthermore, it has been demonstrated that there is a cost-effectiveness of self-management of BP for hypertensive patients with DM and 
other diseases. ${ }^{44}$ Previous studies have reported that hyperuricemia is closely related to the occurrence and progression of kidney disease in the general population and CKD patients. ${ }^{45,46}$ Similarly, the present cohort, in a special population who are at high risk for $\mathrm{KD}$, observes that UA has good predictive value for the occurrence of $\mathrm{KD}$, indicating that UA should be another important marker for the prediction and treatment of KD in this patient group.

Although several predictive tools have been established for kidney disease prediction, few models are specifically applicable to patients with both hypertension and GMD. In addition, some of them need more cost-effective factors and are not quantifiable, which limits the generalization of the tools. The present study develops a simple and practical nomogram for hypertensive patients with GMD, in which the included factors are common and easy-to-obtain in clinical setting and even in primary care setting. Moreover, the prediction model shows good performance by internal validation. Early-stage kidney diseases are usually asymptomatic and is often ignored. ${ }^{3}$ Most of the patients detected for kidney disease are already in an irreversible stage at the time of detection, which leads to the occurrence of complications, and some of them have even progressed to end-stage renal disease requiring renal replacement therapy. Moreover, patients with CKD tended to develop treatment-resistant hypertension and have a higher risk of adverse outcomes. ${ }^{47}$ The present nomogram might help clinicians, and even primary care providers, identify individuals who are at high risk of developing $\mathrm{KD}$, and therefore pay close attention and take prevention measures.

The present study has several strengths. We developed a nomogram using easy-to-obtain parameters for predicting incident KD quantitatively in a specific patient group at high-risk of KD. Moreover, our study contains a high-risk population with longitudinal observation, in which data are obtained from hospital electronic system rather than selfreported, making the results reliable. Several limitations warrant discussion. First, although most of the conventional factors of KD were included, we failed to collect some other baseline information, such as cardiac function, homocysteine, thyroid hormones, et al, which have been reported to be associated with kidney disease ${ }^{48-50}$ However, simple/ fewer variables facilitate the application of predictive models. In addition, because of the lack of urine test, we were only able to report KD defined by eGFR, rather than CKD as strictly defined. Second, the study is conducted in a single center, although in a regional center for hypertension with patients of large age range and ethnic groups and referred by primary care providers from all parts of the region. In addition, the small number of the other ethnic group limits further classification; future studies are needed to verify these in detail. Third, we failed to perform an external validation, although the robustness of the prediction model was verified internally. Thus, the generality of the prediction model needs to be further verified for those in other countries/regions. Fourth, although no significant difference was observed between the study population and the loss to follow-up population in most baseline characteristics, the rate of loss to follow-up (19.3\%) may potentially bias the results.

In conclusion, we identified six factors, which are closely associated with the occurrence of KD among patients with both hypertension and GMD, and developed a simple and practical nomogram for predicting individuals' 5-year risk of incident KD. The model has good accuracy for prediction and shows good performance in internal validation. This quantitative prediction tool could help clinicians, and even primary care providers, recognize potential KD patients early and make strategy for prevention and management.

\section{Data Sharing Statement}

The datasets are available from the corresponding author upon reasonable request.

\section{Ethical Approval}

The present study was approved by the Ethics Committee at the People's Hospital of Xinjiang Uygur Autonomous Region (IRB approval number: 2020020513). All participants or their legal representatives signed written consent forms.

\section{Acknowledgments}

The authors are very much grateful to all study participants for their kind cooperation/participation in the study.

\section{Funding}

This work is partly funded by the National Key R\&D Program of China (grant number: 2018YFC1311503). 


\section{Disclosure}

The authors have declared that no conflict of interest exists.

\section{References}

1. Herzog CA, Asinger RW, Berger AK, et al. Cardiovascular disease in chronic kidney disease. A clinical update from Kidney Disease: Improving Global Outcomes (KDIGO). Kidney Int. 2011;80(6):572-586. doi:10.1038/ki.2011.223

2. Abubakar II, Tillmann T, Banerjee A. Global, regional, and national age-sex specific all-cause and cause-specific mortality for 240 causes of death, 1990-2013: a systematic analysis for the Global Burden of Disease Study 2013. Lancet. 2015;385(9963):117-171. doi:10.1016/S0140-6736(14) 61682-2

3. Jha V, Garcia-Garcia G, Iseki K, et al. Chronic kidney disease: global dimension and perspectives. Lancet. 2013;382(9888):260-272. doi:10.1016/ S0140-6736(13)60687-X

4. Hill NR, Fatoba ST, Oke JL, et al. Global prevalence of chronic kidney disease - a systematic review and meta-analysis. PLoS One. 2016;11(7): e0158765. doi:10.1371/journal.pone.0158765

5. James SL, Abate D, Abate KH, et al. Global, regional, and national incidence, prevalence, and years lived with disability for 354 diseases and injuries for 195 countries and territories, 1990-2017: a systematic analysis for the Global Burden of Disease Study 2017. Lancet. 2018;392 (10159):1789-1858. doi:10.1016/S0140-6736(18)32279-7

6. Lv JC, Zhang LX. Prevalence and disease burden of chronic kidney disease. Adv Exp Med Biol. 2019;1165:3-15.

7. Cao Y, Li W, Yang G, et al. Diabetes and hypertension have become leading causes of CKD in Chinese elderly patients: a comparison between 1990-1991 and 2009-2010. Int Urol Nephrol. 2012;44(4):1269-1276. doi:10.1007/s11255-012-0194-0

8. Wilson S, Mone P, Jankauskas SS, et al. Chronic kidney disease: definition, updated epidemiology, staging, and mechanisms of increased cardiovascular risk. J Clin Hypertens. 2021;23(4):1269-1276. doi:10.1111/jch.14186

9. Ohta M, Babazono T, Uchigata Y, et al. Comparison of the prevalence of chronic kidney disease in Japanese patients with type 1 and type 2 diabetes. Diabet Med. 2010;27(9):1017-1023. doi:10.1111/j.1464-5491.2010.03049.x

10. Huang YM, Xu D, Long J, et al. Spectrum of chronic kidney disease in China: a national study based on hospitalized patients from 2010 to 2015. Nephrology. 2019;24(7):725-736. doi:10.1111/nep.13489

11. Wang L, Peng W, Zhao Z, et al. Prevalence and treatment of diabetes in China, 2013-2018. JAMA. 2021;326(24):2498-2506. doi:10.1001/ jama.2021.22208

12. Cai X, Zhang Y, Li M, et al. Association between prediabetes and risk of all cause mortality and cardiovascular disease: updated meta-analysis. BMJ. 2020;370:m2297. doi:10.1136/bmj.m2297

13. Cai X, Liu X, Sun L, et al. Prediabetes and the risk of heart failure: a meta-analysis. Diabetes Obes Metab. 2021;23(8):1746-1753. doi:10.1111/ dom. 14388

14. Shu J, Matarese A, Santulli G. Diabetes, body fat, skeletal muscle, and hypertension: the ominous chiasmus? J Clin Hypertens. 2019;21(2):239242. doi: $10.1111 /$ jch. 13453

15. Lin M, Heizhati M, Wang L, et al. Prevalence and associated factors of kidney dysfunction in patients with hypertension and/or diabetes mellitus from a primary care population in Northwest China. Int J Gen Med. 2021;14:7567-7578. doi:10.2147/IJGM.S329604

16. Rymarz A, Romejko K, Matyjek A, et al. Serum osteoprotegerin is an independent marker of metabolic complications in non-dialysis dependent chronic kidney disease patients. Nutrients. 2021;13(10):10. doi:10.3390/nu13103609

17. Dounousi E, Tellis C, Pavlakou P, et al. Association between PCSK9 levels and markers of inflammation, oxidative stress, and endothelial dysfunction in a population of nondialysis chronic kidney disease patients. Oxid Med Cell Longev. 2021;2021:6677012. doi:10.1155/2021/6677012

18. Carnagarin R, Nolde JM, Ward NC, et al. Homocysteine predicts vascular target organ damage in hypertension and may serve as guidance for firstline antihypertensive therapy. $J$ Clin Hypertens. 2021;23(7):1380-1389. doi:10.1111/jch.14265

19. Singh TK, Arya V, Navaratnarajah N. Chronic kidney disease and cardiovascular disease: a focus on primary care. Cardiovasc Hematol Disord Drug Targets. 2014;14(3):212-218. doi:10.2174/1871529X14666140401115110

20. Yang L, Chu TK, Lian J, et al. Individualised risk prediction model for new-onset, progression and regression of chronic kidney disease in a retrospective cohort of patients with type 2 diabetes under primary care in Hong Kong. BMJ Open. 2020;10(7):e035308. doi:10.1136/bmjopen2019-035308

21. Gurudas S, Nugawela M, Prevost AT, et al. Development and validation of resource-driven risk prediction models for incident chronic kidney disease in type 2 diabetes. Sci Rep. 2021;11(1):13654. doi:10.1038/s41598-021-93096-w

22. Shih CC, Lu CJ, Chen GD, et al. Risk prediction for early chronic kidney disease: results from an adult health examination program of 19,270 individuals. Int J Environ Res Public Health. 2020;17(14):14. doi:10.3390/ijerph17144973

23. Chien KL, Lin HJ, Lee BC, et al. A prediction model for the risk of incident chronic kidney disease. Am J Med. 2010;123(9):836-46.e2. doi:10.1016/j.amjmed.2010.05.010

24. Winiarska A, Knysak M, Nabrdalik K, et al. Inflammation and oxidative stress in diabetic kidney disease: the targets for SGLT2 inhibitors and GLP-1 receptor agonists. Int J Mol Sci. 2021;22(19):10822. doi:10.3390/ijms221910822

25. Consultation. WHO. Definition and diagnosis of diabetes and intermediate hyperglycaemia; 2006.

26. Levey AS, Stevens LA, Schmid CH, et al. A new equation to estimate glomerular filtration rate. Ann Intern Med. 2009;150(9):604-612. doi:10.7326/0003-4819-150-9-200905050-00006

27. Collins GS, Reitsma JB, Altman DG, et al. Transparent reporting of a multivariable prediction model for individual prognosis or diagnosis (TRIPOD): the TRIPOD statement. BMJ. 2015;350:g7594. doi:10.1136/bmj.g7594

28. Alhamzawi R, Ali HTM. The Bayesian adaptive lasso regression. Math Biosci. 2018;303:75-82. doi:10.1016/j.mbs.2018.06.004

29. Sauerbrei W, Royston P, Binder H. Selection of important variables and determination of functional form for continuous predictors in multivariable model building. Stat Med. 2007;26(30):5512-5528. doi:10.1002/sim.3148

30. Tannor EK, Sarfo FS, Mobula LM, et al. Prevalence and predictors of chronic kidney disease among Ghanaian patients with hypertension and diabetes mellitus: a multicenter cross-sectional study. J Clin Hypertens. 2019;21(10):1542-1550. doi:10.1111/jch.13672 
31. Lin YS, Liu PH, Lin SW, et al. Simple obstructive sleep apnea patients without hypertension or diabetes accelerate kidney dysfunction: a population follow-up cohort study from Taiwan. Sleep Breath. 2017;21(1):85-91. doi:10.1007/s11325-016-1376-2

32. Koye DN, Shaw JE, Reid CM, et al. Incidence of chronic kidney disease among people with diabetes: a systematic review of observational studies. Diabet Med. 2017;34(7):887-901. doi:10.1111/dme.13324

33. Huang Y, Cai X, Mai W, et al. Association between prediabetes and risk of cardiovascular disease and all cause mortality: systematic review and meta-analysis. BMJ. 2016;355:i5953. doi:10.1136/bmj.i5953

34. Mai L, Wen W, Qiu M, et al. Association between prediabetes and adverse outcomes in heart failure. Diabetes Obes Metab. 2021;23(11):24762483. doi:10.1111/dom. 14490

35. Ji A, Pan C, Wang H, et al. Prevalence and associated risk factors of chronic kidney disease in an elderly population from Eastern China. Int $J$ Environ Res Public Health. 2019;16(22):4383. doi:10.3390/ijerph16224383

36. Wang F, He K, Wang J, et al. Prevalence and risk factors for CKD: a comparison between the adult populations in China and the United States. Kidney Int Rep. 2018;3(5):1135-1143. doi:10.1016/j.ekir.2018.05.011

37. Heizhati M, Wang L, Yao X, et al. Prevalence, awareness, treatment and control of hypertension in various ethnic groups (Hui, Kazakh, Kyrgyz, Mongolian, Tajik) in Xinjiang, Northwest China. Blood Press. 2020;29(5):276-284. doi:10.1080/08037051.2020.1745055

38. Wang Z, Chen Z, Zhang L, et al. Status of hypertension in China: results from the China hypertension survey, 2012-2015. Circulation. 2018;137 (22):2344-2356. doi:10.1161/CIRCULATIONAHA.117.032380

39. Tohidi M, Hasheminia M, Mohebi R, et al. Incidence of chronic kidney disease and its risk factors, results of over 10 year follow up in an Iranian cohort. PLoS One. 2012;7(9):e45304. doi:10.1371/journal.pone.0045304

40. Al-Shamsi S, Oulhaj A, Regmi D, et al. Use of estimated glomerular filtration rate to predict incident chronic kidney disease in patients at risk of cardiovascular disease: a retrospective study. BMC Nephrol. 2019;20(1):325. doi:10.1186/s12882-019-1494-8

41. Taal MW, Brenner BM. Predicting initiation and progression of chronic kidney disease: developing renal risk scores. Kidney Int. 2006;70 (10):1694-1705. doi:10.1038/sj.ki.5001794

42. Hemmelgarn BR, Zhang J, Manns BJ, et al. Progression of kidney dysfunction in the community-dwelling elderly. Kidney Int. 2006;69(12):21552161. doi: $10.1038 /$ sj.ki.5000270

43. Eriksen BO, Stefansson VT, Jenssen TG, et al. Blood pressure and age-related GFR decline in the general population. BMC Nephrol. 2017;18 (1):77. doi:10.1186/s12882-017-0496-7

44. Penaloza-Ramos MC, Jowett S, Mant J, et al. Cost-effectiveness of self-management of blood pressure in hypertensive patients over 70 years with suboptimal control and established cardiovascular disease or additional cardiovascular risk diseases (TASMIN-SR). Eur J Prev Cardiol. 2016;23 (9):902-912. doi:10.1177/2047487315618784

45. Oh TR, Choi HS, Kim CS, et al. Hyperuricemia has increased the risk of progression of chronic kidney disease: propensity score matching analysis from the KNOW-CKD study. Sci Rep. 2019;9(1):6681. doi:10.1038/s41598-019-43241-3

46. Lin B, Shao L, Luo Q, et al. Prevalence of chronic kidney disease and its association with metabolic diseases: a cross-sectional survey in Zhejiang province, Eastern China. BMC Nephrol. 2014;15(1):36. doi:10.1186/1471-2369-15-36

47. Thomas G, Xie D, Chen HY, et al. Prevalence and prognostic significance of apparent treatment resistant hypertension in chronic kidney disease: report from the chronic renal insufficiency cohort study. Hypertension. 2016;67(2):387-396. doi:10.1161/HYPERTENSIONAHA.115.06487

48. Xie D, Yuan Y, Guo J, et al. Hyperhomocysteinemia predicts renal function decline: a prospective study in hypertensive adults. Sci Rep. 2015;5 (1):16268. doi:10.1038/srep16268

49. Jankauskas SS, Morelli MB, Gambardella J, et al. Thyroid hormones regulate both cardiovascular and renal mechanisms underlying hypertension. $J$ Clin Hypertens. 2021;23(2):373-381. doi:10.1111/jch.14152

50. Wu J, Zheng H, Liu X, et al. Prognostic value of secreted frizzled-related protein 5 in heart failure patients with and without type 2 diabetes mellitus. Circ Heart Fail. 2020;13(9):e007054. doi:10.1161/CIRCHEARTFAILURE.120.007054

\section{Publish your work in this journal}

Risk Management and Healthcare Policy is an international, peer-reviewed, open access journal focusing on all aspects of public health, policy, and preventative measures to promote good health and improve morbidity and mortality in the population. The journal welcomes submitted papers covering original research, basic science, clinical \& epidemiological studies, reviews and evaluations, guidelines, expert opinion and commentary, case reports and extended reports. The manuscript management system is completely online and includes a very quick and fair peer-review system, which is all easy to use. Visit http://www.dovepress.com/testimonials.php to read real quotes from published authors.

Submit your manuscript here: https://www.dovepress.com/risk-management-and-healthcare-policy-journal 